\title{
Innovative medical technologies in the percutaneous treatment of tricuspid regurgitation in Poland
}

\author{
Adam Witkowski ${ }^{1}$, Dariusz Dudek ${ }^{2,3}$, Stanisław Bartuśs ${ }^{2}$, Wojciech Wojakowski ${ }^{4}$, \\ Andrzej Gackowski ${ }^{5}$, Marek Grygier ${ }^{6}$, Mariusz Kuśmierczyk ${ }^{7}$, Miłosz J. Jaguszewski ${ }^{8}$, \\ Ewa Kowalik $^{9}$, Katarzyna Bondaryk ${ }^{10}$, Maciej Niewada ${ }^{11,12}$, \\ Piotr Przygodzki ${ }^{13}$, Michał Jakubczyk ${ }^{12,14}$
}

${ }^{1}$ Department of Cardiology and Interventional Angiology, National Institute of Cardiology, Warsaw, Poland; ${ }^{2}$ Institute of Cardiology, Jagiellonian University Medical College, Krakow, Poland; ${ }^{3}$ Maria Cecilia Hospital, GVM Care and Research, Cotignola (RA), Ravenna, Italy; ${ }^{4}$ Division of Cardiology and Structural Heart Diseases, SMK in Katowice, Poland; ${ }^{5}$ Jagiellonian University, Medical College, Institute of Cardiology, Department of Coronary Disease and Heart Failure, Noninvasive Cardiovascular Laboratory, John Paul II Hospital, Krakow, Poland; ${ }^{6}$ First Department of Cardiology, Poznan University of Medical Sciences, Poznan, Poland; ${ }^{7}$ Department of Cardiac Surgery and Transplantology, National Institute of Cardiology, Warsaw, Poland; ${ }^{8}$ First Department of Cardiology, Medical University of Gdansk, Poland; ${ }^{9}$ Department of Congenital Heart Diseases, National Institute of Cardiology, Warsaw, Poland; ${ }^{10}$ Law Office Bondaryk, Warsaw, Poland; ${ }^{11}$ Department of Experimental and Clinical Pharmacology, Medical University of Warsaw, Poland; ${ }^{12}$ HealthQuest, Poland; ${ }^{13}$ Abbott Medical sp. $z$ o.o., Health Economics and Reimbursement, Warsaw, Poland; ${ }^{14}$ SGH Warsaw School of Economics, Poland

\section{The paper was guest edited by Prof. Dariusz Jagielak}

\begin{abstract}
Tricuspid regurgitation (TR) usually develops secondarily to left-sided heart diseases, whereas primary lesions to the valve apparatus is less common. Untreated severe TR has a poor prognosis and surgical treatment, i.e., valve repair or replacement, is the only treatment option with class I recommendation. However, cardiac surgical procedures may be associated with a high risk of complications. Recent advances in percutaneous approaches to managing structural heart diseases, especially mitral valve diseases, have enabled the implementation of this therapeutic strategy in the population of patients with $T R$. This paper presents data on the clinical efficacy, cost-effectiveness and expected population size for one of these procedures, namely the TriClip TTVr System procedure. Its efficacy was assessed in the TRILUMINATE study involving 85 patients with co-morbidities and at high surgical risk. After 1 year of follow-up, the reduction in the TR grade was reported in $71 \%$ of patients. Clinical improvement in New York Heart Association functional class, a 6-minute walk test, and the quality of life were also observed. A published analysis comparing percutaneous treatment modalities with a drug therapy based on data from medical registers was utilized, and propensity score matching was also employed. Percutaneous treatment reduced 1-year mortality and rehospitalization risk. The economic analysis showed the use of TriClip TTVr System is cost-effective: the cost of an additional quality-adjusted life year ranged from approximately PLN 85,000 to PLN 100,000, which is below the official threshold in Poland. The potential annual number of candidates for this treatment modality in Poland is estimated at 265. (Cardiol J 2022; 29, 3: 369-380)
\end{abstract}

Key words: tricuspid regurgitation, transcatheter tricuspid valve interventions, transcatheter tricuspid valve repair, TriClip TTVr System, MitraClip

Address for correspondence: Dr. Maciej Niewada, Department of Experimental and Clinical Pharmacology, Medical University of Warsaw, ul. Banacha 1b, 02-097 Warszawa, Poland, tel: +48 22116 61 60, fax: +48 221166202 , e-mail: maciej.niewada@wum.edu.pl

Received: 19.07.2021 Accepted: 6.10.2021 Early publication date: 18.10.2021

This article is available in open access under Creative Common Attribution-Non-Commercial-No Derivatives 4.0 International (CC BY-NC-ND 4.0) license, allowing to download articles and share them with others as long as they credit the authors and the publisher, but without permission to change them in any way or use them commercially. 


\section{Introduction}

This paper summarizes the discussion held at meetings of an advisory board comprised of experts in interventional cardiology and cardiac surgery. The meetings took place between May and September 2020. They were attended by 7 clinicians representing 5 centers experienced in treating patients with tricuspid regurgitation (TR). This paper aims to identify a potential place for CE-marked TriClip TTVr System (Abbott, USA; Central illustration) in the treatment of TR in Poland, present clinical evidence of its effectiveness, attempt to determine its cost-effectiveness and estimate the number of potential Polish patients if the technology was available.

\section{Tricuspid regurgitation: Epidemiology}

Tricuspid regurgitation is the most common heart valve pathology. Trace or mild TR is a common finding, reported in over $70 \%$ of the population [1]. In a study published in 2020 which included more than 33,000 patients referred for echocardiography, mild TR was detected in $21.9 \%$ of patients, moderate $-8 \%$, while severe $-0.8 \%$ [2]. OxVALVE registry showed that prevalence of moderate/severe TR in the general population was $2.7 \%$ [3].

The incidence of TR increases with age. In a population-based study conducted in Olmsted County (USA), moderate or severe TR was detected in $0.05 \%$ of patients aged $18-44$ years and in $3.96 \%$ of patients aged $>75$ years [4]. TR was significantly more frequently diagnosed in females. Similar relationships were presented in an earlier analysis based on the Framingham study: in the 40-49-year age group, at least moderate TR was detected in $0.5 \%$ of females and $0.3 \%$ of males while in the $70-83$-year age group - in $5.6 \%$ of females and $1.5 \%$ of males [1]. Therefore, it can be assumed that moderate-to-severe TR affects approximately $4 \%$ of individuals over 75 years of age. Isolated regurgitation was diagnosed in about $8 \%$ of patients, while others had co-existent cardiac and non-cardiac comorbidities.

No reliable data on the incidence of TR in Poland are available. Given the epidemiological data for the United States, it is estimated that some level of TR affects approximately 200,000 individuals in Poland [5].

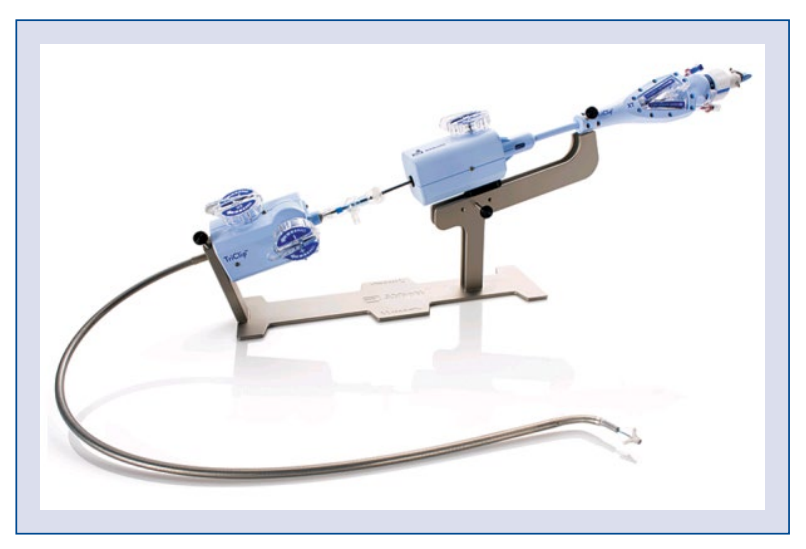

Central illustration. TriClip XT Delivery System.

\section{Etiology and pathogenesis of tricuspid regurgitation}

Most TR cases are secondary to right ventricular (RV) overload and dilation. The most common mechanism underlying TR development is the dilation of the valve annulus (at the site of insertion of the anterior and posterior leaflets) with normal leaflet morphology (type I) and/or RV enlargement (which results in papillary muscle displacement with restricted leaflet position) - type IIIb. Secondary TR occurs due to left-sided heart diseases (including left ventricular [LV] dysfunction and mitral valve disease), other forms of pulmonary hypertension, atrial fibrillation, heart tumors or as a result of RV myocardial injury in the course of coronary artery disease or cardiomyopathy.

Injury to valve leaflets or the subvalvular apparatus, which leads to the development of the much less common primary TR $(\sim 15 \%)$ [4], may be caused by infective endocarditis (especially in cases of intravenous drug abuse), rheumatic heart disease, carcinoid syndrome, myxoid degeneration, endomyocardial fibrosis, congenital defects (Ebstein's anomaly, congenital valve dysplasia), chest trauma or the increasingly common iatrogenic injury (transvenous electrodes, endomyocardial biopsy, ionizing radiation, drug-induced lesions).

Tricuspid regurgitation leads to right atrial and RV volume overload; once the compensatory capacity of the right heart chambers is exhausted, the patient develops systemic venous congestion and severe heart failure. 


\section{Clinical presentation and prognosis of patients with tricuspid regurgitation}

Clinical manifestations of this defect depend on the severity of the comorbidities and initially tend to be non-specific (fatigue, worsened exercise tolerance, shortness of breath). Patients develop peripheral edema, jugular vein dilatation and hepatomegaly. Persistent, hemodynamically significant TR results in increased venostasis and edema (despite diuretic therapy) as well as hepatic hyperemia, which lead to organ dysfunction and gradual development of cardiac cirrhosis.

The diagnosis of advanced TR worsens the prognosis. A meta-analysis of 70 studies involving over 32,500 patients with a mean follow-up of $3.2 \pm 2.1$ years showed that moderate/severe TR was associated with a 2 -fold increase in the risk of death compared with mild/no regurgitation (relative risk $[\mathrm{RR}]=1.95 ; 95 \%$ confidence interval $[\mathrm{CI}]$ $=1.75-2.17$ ) [6]. The relationship between TR severity and all-cause mortality remained significant also after adjustment for pulmonary artery systolic pressure (PASP; meta-analysis of 13 studies; RR $=1.85 ; 95 \% \mathrm{CI}=1.44-2.39)$ and $\mathrm{RV}$ dysfunction (meta-analysis of 15 studies; $\mathrm{RR}=1.78 ; 95 \% \mathrm{CI}$ $=1.49-2.13)$. Moderate/severe TR also increased cardiac mortality $(\mathrm{RR}=2.56 ; 95 \% \mathrm{CI}=1.84-3.55)$ and heart failure hospitalization rates $(\mathrm{RR}=1.73$; $95 \% \mathrm{CI}=1.14-2.62)$. A gradual increase in the risk of all-cause mortality was observed $(\mathrm{RR}=1.25$; 1.61; and 3.44, respectively; $p<0.001$ for the trend) in patients with mild, moderate and severe regurgitation compared to patients without TR.

The presence of significant TR worsens the prognosis of patients regardless of LV function. A large retrospective study (over 5,200 patients, 4-year follow-up) showed that moderate or highergrade TR was associated with increased mortality regardless of PASP (hazard ratio $[\mathrm{HR}]=1.31$; $95 \% \mathrm{CI}=1.16-1.49$ for PASP $>40 \mathrm{mmHg}$ and $\mathrm{HR}=1.32,95 \% \mathrm{CI}=1.05-1.62$ for $\leq 40 \mathrm{mmHg}$ ) and regardless of $\mathrm{LV}$ ejection fraction $(\mathrm{EF})(\mathrm{HR}=1.49$; $95 \% \mathrm{CI}=1.34-1.66$ for $\mathrm{EF}<50 \%$ and $\mathrm{HR}=1.54$; $95 \% \mathrm{CI}=1.37-1.71$ for $\mathrm{EF} \geq 50 \%$ ) [7]. Compared with the absence of TR, patients with moderateto-severe defects had worse prognosis also after adjustment for age, systolic function of both ventricles, RV dimension and inferior vena cava dilatation (HR 1.17; 95\% CI = 0.96-1.42 for moderate regurgitation and $\mathrm{HR}=1.31 ; 95 \% \mathrm{CI}=1.05-1.66$ for severe regurgitation).

\section{Diagnostic evaluation}

Transthoracic echocardiography (and transesophageal echocardiography in selected cases) is the most important diagnostic method in evaluation of TR prior to intervention. It requires advanced skills and high-quality equipment utilizing full range of echocardiographic techniques, including 3-dimensional (3D) imaging. Echocardiography plays a key role in differentiating between primary and secondary valve regurgitation for determining the main mechanism and etiology of the defect and assessing its severity. The evaluation should involve assessment of the morphology of the valve itself, the size of the right heart chambers and tricuspid annulus, dimension and collapsibility of the inferior vena cava and assessment of RV systolic function. Doppler analysis of the regurgitation jet and the assessment of the RV systolic pressure are also crucial elements of comprehensive assessment. Despite the use of new echocardiographic techniques (including 3D imaging), valve assessment is challenging in some patients because of poor echogenicity of leaflets and difficulties in obtaining a good acoustic window. Special skills are required to carry out transesophageal assessment because, in contrast to mitral valve, tricuspid valve is located distally and non-axially to the oesophagus.

Tricuspid regurgitation grading is based on a combined analysis of multiple parameters [8]: qualitative, semi-quantitative and quantitative. Qualitative criteria apply to valve morphology (leaflet thickening/prolapse/restriction/substantial coaptation defect), regurgitation jet assessment in color Doppler (very large central jet or eccentric jet striking the right atrial wall) and assessment of the regurgitation jet by continuous wave Doppler signal (dense/triangular with early peaking [peak velocity of $<2.0 \mathrm{~m} / \mathrm{s}$ in severe TR]). Semi-quantitative criteria are as follows:

- regurgitation vena contracta width $\geq 7 \mathrm{~mm}$;

- proximal isovelocity surface area radius $\geq 9 \mathrm{~mm}$ at Nyquist cutoff value of $0.3 \mathrm{~m} / \mathrm{s}$;

- hepatic veins systolic flow reversal;

- tricuspid inflow pattern with $\mathrm{E}$ wave dominance $\geq 1.0 \mathrm{~m} / \mathrm{s}$.

Echocardiographic quantitative criteria for severe TR include:

- effective regurgitant orifice area (EROA) $\geq 40 \mathrm{~mm}^{2}$;

- regurgitation jet volume $\geq 45 \mathrm{~mL}$;

- enlargement of the right heart chambers, and dilatation of the inferior vena cava. 
It should be noted that so far, no cut-off points for moderate and mild regurgitation have been established for quantitative parameters of TR (EROA, regurgitation jet volume). However, as demonstrated by clinical studies of new percutaneous valve repair techniques, severe TR affects patients with very advanced defects and regurgitation jet quantitative parameters significantly above the cutoffs proposed. To allow for precise grading of TR in this group of patients in the context of enrolment for percutaneous interventions and their outcome monitoring, Hahn and Zamorano [9] presented a new, 5 -grade classification of TR. They proposed additional massive regurgitation and torrential regurgitation based on the assessment of vena contracta (14-20 $\mathrm{mm}$ for massive regurgitation and $\geq 21 \mathrm{~mm}$ for torrential regurgitation) and EROA (60-79 $\mathrm{mm}^{2}$ for massive regurgitation and $\geq 80 \mathrm{~mm}^{2}$ for torrential regurgitation). Furthermore, they introduced another echocardiographic assessment criterion, namely $3 \mathrm{D}$ vena contracta area with the cutoffs of $75-94 \mathrm{~mm}^{2}$ for severe regurgitation, $95-114 \mathrm{~mm}^{2}$ for massive regurgitation, and $\geq 115 \mathrm{~mm}^{2}$ for torrential regurgitation. This expanded TR classification also has a prognostic value, because the outcomes of patients with massive and torrential TR have incremental risk of mortality in comparison to severe TR.

In turn, Dreyfus et al. [10] proposed classification of functional TR and qualification for surgical treatment based not on regurgitation jet assessment alone but also on the tricuspid annular size (diastolic diameter of $>40 \mathrm{~mm}$ is considered a significant dilatation) and tricuspid leaflet coaptation (normal/leaflet restriction/no coaptation).

\section{Current recommendations of scientific societies on the management and treatment of tricuspid regurgitation}

Tricuspid regurgitation is a major challenge not only in terms of diagnostics but primarily in terms of treatment. Currently, surgical treatment is the standard of care in severe symptomatic isolated valve regurgitation. In the presence of other left-sided valve defects requiring surgery, indications for tricuspid valve repair include severe TR or moderate TR with coexistent tricuspid annular dilatation.

Several valve repair techniques have been proposed for cardiac surgery for TR [11]. The first method, introduced by Kay et al. [12], involves posterior leaflet plication, which leads to the formation of the bicuspid tricuspid valve. Subsequently, de
Vega [13] introduced tricuspid valve annuloplasty with suture placement in the posterior and anterior part of the annulus (bypassing atrioventricular node). Another modification of surgery was annuloplasty using a synthetic ring that restores the normal shape of the valve. Insertion of a semi-open rigid ring is currently the most widely used method of surgical tricuspid valve repair.

In cases of RV enlargement and remodeling with leaflet restriction, annular reduction as the only treatment option may not be effective enough. In such a situation, anterior tricuspid leaflet extension or the clover technique is applied, i.e., suturing valve leaflet edges (similar to the Alfieri technique for mitral valve).

In severe leaflet restriction or organic lesions preventing effective repair, artificial (mechanical or biological) valve implantation is necessary. Biological valves are preferred due to a lower risk of thrombosis and possibly the chances of subsequent percutaneous treatment of its dysfunction (valve-in-valve procedure).

According to the 2017 guidelines of the European Society of Cardiology (ESC) and the European Association for Cardiothoracic Surgery (EACTS) on the treatment of valvular heart defects [14], a surgical procedure is indicated (class I) in patients with severe primary or secondary TR undergoing left heart defect surgery. Surgery should also be considered (class IIa recommendation) in patients with moderate primary TR undergoing left heart surgery, as well as in patients with mild to moderate secondary valve insufficiency with tricuspid annulus dilatation $\left(\geq 40 \mathrm{~mm}\right.$ or $>21 \mathrm{~mm} / \mathrm{m}^{2}$, evaluated using $2 \mathrm{D}$ echocardiography) undergoing left heart surgery. Surgical treatment may be considered at a time of left heart surgery (class IIb recommendation) in patients with a history of right-sided heart failure and mild-to-moderate secondary TR, even if the tricuspid valve annulus is not dilated.

Indications for surgical treatment of isolated TR include symptomatic disease with severe isolated primary TR without severe RV dysfunction (class I recommendation). Surgery should be considered in asymptomatic patients or patients with mild symptoms with severe primary TR and progressive RV dilatation or worsening RV function (class IIa). After surgery for left heart defects and in the absence of recurrent left valve dysfunction, surgery should be considered in patients with severe secondary tricuspid insufficiency who develop symptoms or progressive RV dilatation/dysfunction, in the absence of severe RV or LV dysfunction and severe pulmonary vascular disease/pulmonary 
hypertension (class IIa). Both ESC/EACTS recommendations on the surgical treatment of TR have the level of evidence $\mathrm{C}$, which means that they are based on an agreed expert opinion or data from small retrospective studies or registries.

Similar recommendations on the interventional treatment of TR were provided by the American Heart Association/American College of Cardiology (AHA/ACC) in 2020 [15]. The recommendations concern valve repair primarily in patients undergoing left heart surgery. As for the surgical treatment of symptomatic patients with severe primary TR, a lower class (IIa) recommendation was provided compared to the European guidelines. In addition, special attention is paid to atrial fibrillation in the USA guidelines as the cause of isolated secondary TR resulting primarily from annular dilatation.

Eligibility for surgical treatment should be based on the patient's clinical status, co-morbidities, and perioperative risk. The right moment for the surgery of the defect has not been determined. Similarly, no optimal drug therapy for TR has been defined. According to the AHA's position statement on the diagnosis and treatment of RV insufficiency of 2018, drug therapy for RV insufficiency should be based on diuretics (or renal replacement therapy, if increased diuretics doses failed); afterload-reducing agents are also used (vasodilators for pulmonary circulation), and in extreme cases, mechanical circulatory support [16]. Late-onset symptoms of TR and delayed qualification for surgical treatment of the defect have a negative impact on treatment outcomes [17]. Early and late outcomes of surgical treatment of TR are still unsatisfactory.

In an analysis of nearly 55,000 tricuspid valve surgeries in the USA between 2000 and 2010 (in $85.7 \%$ of cases, the procedure on tricuspid valve accompanied other procedures, with $88.9 \%$ of valve repairs), the perioperative mortality rate was $10.6 \%$ in 2000 and $8.2 \% 10$ years later [18]. Predictors of mortality included: older age, high creatinine level, renal failure requiring dialysis prior to surgery, cardiogenic shock, use of intra-aortic counterpulsation or inotropic agents, presence of significant peripheral or cerebrovascular disease, mitral stenosis, myocardial infarction, tricuspid valve replacement, chronic pulmonary disease, diabetes mellitus, coronary artery disease, urgent surgery, reoperation, congestive heart failure, and all other procedures except for double valve replacement (mitral or aortic).

More recent data (2007-2017) on the outcomes of surgical treatment of isolated TR come from 12 centers in France [19]. Most of the 466 patients who underwent surgery for tricuspid valve alone (8\% of all TR surgeries) had an advanced disease (47\% New York Heart Association [NYHA] class II/IV; $57 \%$ with symptoms of right-ventricular failure). Of these patients, $49 \%$ were diagnosed with functional TR (22\% after left heart surgery, $27 \%$ isolated TR) and $51 \%$ had an organic defect (including $31 \%$ with infective endocarditis). Overall, inhospital mortality following surgery for isolated TR was $10 \%$, including $16 \%$ of patients after left heart surgery, $13 \%$ of patients with isolated functional TR, $5 \%$ of patients with infective endocarditis and $8 \%$ of patients with organic TR of another etiology. A multivariate analysis showed that independent risk factors for in-hospital mortality were as follows: NYHA class III/IV (odds ratio $[\mathrm{OR}]=2.7$; $95 \% \mathrm{CI}=1.2-6.1 ; \mathrm{p}=0.01)$, moderate/severe RV dysfunction $(\mathrm{OR}=2.6 ; 95 \% \mathrm{CI}=1.2-5.8 ; \mathrm{p}=0.02)$ and lower prothrombin time $(\mathrm{OR}=0.98 ; 95 \% \mathrm{CI}=$ $0.96-0.99 ; p=0.008)$; clinical signs of heart failure fell just short of statistical significance $(\mathrm{OR}=2.4$; $95 \% \mathrm{CI}=0.9-6.5 ; \mathrm{p}=0.06)$. Overall, the 1 -year survival rate following surgery for isolated TR was $86 \%$, while the 5 -year survival rate was $75 \%$. Independent predictors of mortality in longer follow-up were as follows: NYHA class III/IV (HR $=1.7 ; 95 \%$ $\mathrm{CI}=1.1-2.8 ; \mathrm{p}=0.04)$, clinical signs of right heart failure $(\mathrm{OR}=1.8 ; 95 \% \mathrm{CI}=1.01-3.3 ; \mathrm{p}<0.05)$, and lower prothrombin time $(\mathrm{OR} 0.98 ; 95 \% \mathrm{CI}=$ $0.97-0.99 ; \mathrm{p}=0.04)$.

\section{New methods of percutaneous tricuspid valve repair}

Minimally invasive surgical procedures and percutaneous valve repair have recently been used to treat valvular heart disease. However, due to technical challenges resulting from the tricuspid annulus structure, the tricuspid valve was called a 'forgotten valve' in terms of the percutaneous treatment of heart defects. Only in recent years have attempts been made at the transcatheter treatment of TR, based on percutaneous mitral valve treatment experience [20].

Percutaneous treatment modalities for TR are based on:

1. Reduction in the tricuspid annular dimension (percutaneous annuloplasty):

a. Cardioband (ValtechCardio) — direct annuloplasty using an anchoring system to fix and then reduce the size of an artificial annulus, technique originally introduced in the percutaneous treatment of mitral regurgitation, 


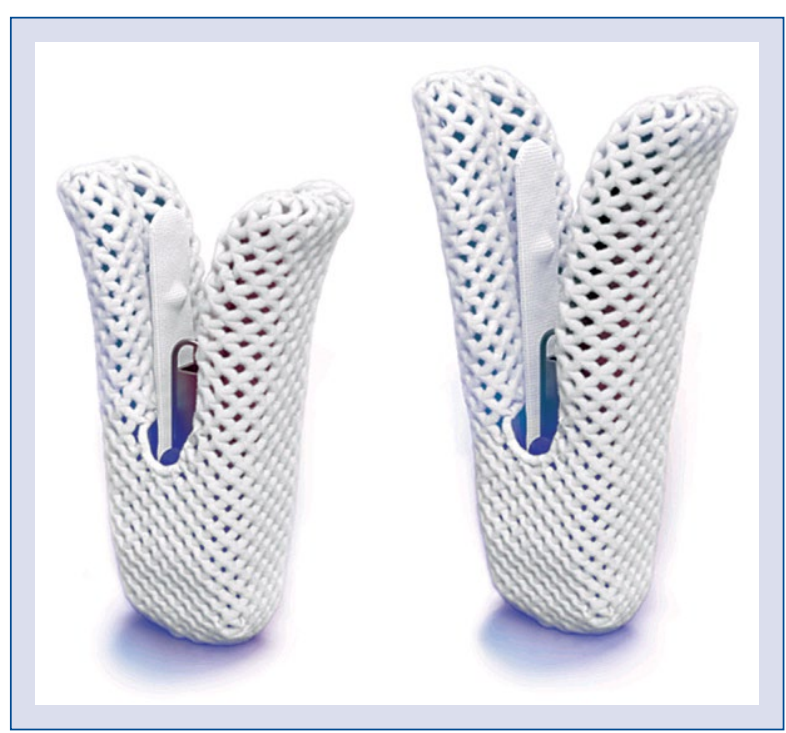

Figure 1. TriClip NT-TriClip XT implant.

b. The Trialign System (Mitralign, Inc.) sutures are placed percutaneously in the commissural area; cinching of the sutures results in plication of the posterior cusp and bicuspidisation of the valve,

c. The TriCinch System (4Tech Cardio) by placing one end of the system in the anteroposterior commissure area and the other in the inferior vena cava, a change in the tricuspid annular geometry is achieved;

2. Improvement of cusp coaptation:

a. The Forma System (Edwards Lifesciences) - a polymer balloon is placed in the tricuspid orifice and anchored in the RV apex. The balloon fills up the regurgitant orifice,

b. The MitraClip System (Abbott Vascular) - a system for percutaneous treatment of mitral valve regurgitation, based on the Alfieri surgical technique (edge-to-edge),

d. The PASCAL System (Edwards Lifesciences) - involving the approximation of valve leaflets, used for percutaneous mitral valve repair,

e. The TriClip TTVr System (Abbott Vascular) — based on the MitraClip System in which the control and clip delivery system were re-designed for the anatomy and morphology of the right atrioventricular valve (Fig. 1), soon to be complemented by a new generation Triclip G4 TTVr System;

3. Caval valve implantation (CAVI): a. Sapien XT and Sapien 3 valves (Edwards Lifesciences) implanted into the inferior vena cava,

b. The TricValve System (P+F PRODUCTS + FEATURES GMBH) based on the implantation of biological valves mounted to nitinol stents into the superior and inferior vena cava;

4. Percutaneous tricuspid valve implantation (to replace dysfunctional bioprosthesis implanted in the tricuspid orifice):

a. The Melody valve (Medtronic),

b. The NAVIGATE valve (NaviGate Cardiac Structures Inc.),

c. The Sapien valve (Edwards Lifesciences);

5. Transcatheter percutaneous implantation of a dedicated self-expanding valve:

a. The Evoque valve (Edwards Lifesciences).

The outcomes of percutaneous treatment of TR were analyzed, among others, using data from the international TRIVALVE registry. The first data concerned 106 patients with severe symptomatic TR undergoing percutaneous interventions at 11 centers in Europe, the USA and Canada between January 2014 and December 2016 [21]. The mean patient age was $76 \pm 9$ years, $60.4 \%$ of patients were females, and the EuroScore (European System for Cardiac Operative Risk Evaluation) II score was $7.6 \pm 5.7 \%$. In total, $35 \%$ of patients had undergone left heart valve surgery. RV dysfunction (defined as tricuspid annular plane systolic excursion $<17 \mathrm{~mm}$ ) was observed in $56.3 \%$ of patients, 95\% were in NYHA class III/IV. In patients who underwent the intervention, functional regurgitation predominated (95.2\%); the tricuspid annular diameter was $45.4 \pm 11 \mathrm{~mm}$, and pre-intervention PASP was $39.7 \pm 13.8 \mathrm{mmHg}$. The MitraClip System was most frequently used $(n=58)$; other devices used were: Trialign $(n=17)$, TriCinch $(n=15)$, FORMA $(n=7)$, Cardioband $(n=5)$, and venae cavae valves $(n=3)$. Procedure efficacy, defined as successful implantation of the device and TR reduction to grade $\leq 2$, was observed in $62 \%$ of patients. In the 30 -day follow-up period, overall mortality was $3.7 \%$ and the incidence of serious cardiovascular and cerebrovascular events was $26 \%$. More than half of the patients $(58 \%)$ were in NYHA class I/II 30 days after the procedure.

Since most patients with TR underwent the 'edge-to-edge' procedure with the use of the MitraClip System, the authors of the TRIVALVE registry summarized the procedure effectiveness at 1 year of follow-up [22]. The analysis included 249 patients with a mean age of $77 \pm 9$ 
(EuroScore II mean score: $6.4 \%$ ). With the use of two clips on average (standard deviation $=1$ ), the procedure was effective in $77 \%$ of patients. At 1 year of follow-up, sustained improvement in the TR grade was observed in $72 \%$ of patients, and $69 \%$ of patients were in NYHA class I/II. Overall, 1 year mortality was $20 \%$, and the composite endpoint of mortality and unscheduled hospitalizations for heart failure was $35 \%$. Predictors of annual mortality were as follows: ineffective procedure, worsening of renal function and absence of sinus rhythm.

Data from the TRIVALVE registry showed that 'edge-to-edge' procedures are effective in most patients and demonstrated marked clinical improvement in high-risk patients subjected to percutaneous TR repair.

\section{The estimated effectiveness of percutaneous tricuspid regurgitation treatment using the MitraClip TMVr and TriClip TTVr Systems}

A systematic review of primary studies of percutaneous TR repair (regardless of the devices used) was conducted. The search strategy allowed for both experimental and observational (including non-comparative) studies and was not limited to specific devices used for valve repair. The review included PubMed, Embase and Cochrane Library data (search cut-off date March 20, 2020).

A total of 58 papers were included in the review-based analysis. One comparative study was found in which TTVr was compared with drug therapy [23], and 1 study concerning TriClip technology - a prospective TRILUMINATE registry [24]. Both studies are discussed below.

\section{Taramasso 2019 study}

The study involved 472 patients included in the TriValve registry, from 22 European and North American sites, who underwent transcatheter tricuspid valve intervention (TTVI) between 2016 and 2018. The control group was comprised of patients from two retrospective registries, including patients with TR who received drug therapy and were additionally included in the analysis.

The study was conducted using propensity score matching - a statistical technique aimed at increasing the comparability of two patient groups in non-randomized trials by dividing patients into pairs based on an estimation of their similarity in terms of clinical parameters, to reduce the impact of potential confounding factors, i.e., factors affecting the choice of treatment method and at the same time its outcome, and thereby estimate the causal effect of the technology in question [25]. Propensity score matching is widely used in clinical analyzes in the absence of randomized studies, including studies of cardiac procedures [26-28].

Propensity score matching yielded 268 patient pairs. The primary endpoint was the annual allcause mortality, heart failure rehospitalization rate, or the composite endpoint of both events.

After matching, patients in the TTVI group, compared with patients receiving drug therapy, had lower 1-year mortality $(23 \pm 3 \%$ vs. $36 \pm 3 \%, p=$ $0.001)$, a lower risk of rehospitalization (27 $\pm 3 \%$ vs. $47 \pm 3 \%, \mathrm{p}<0.0001)$ and a lower risk of the composite endpoint $(32 \pm 4 \%$ vs. $49 \pm 3 \%, \mathrm{p}=$ 0.0003). The survival analysis using the Cox model showed that TTVI was associated with a reduction in the risk of composite endpoint $(\mathrm{HR}=0.60 ; 95 \%$ $\mathrm{CI}=0.46-0.79 ; \mathrm{p}=0.003$ ), also in the multivariate analysis, after adjustment for gender, NYHA class, $\mathrm{RV}$ dysfunction, and atrial fibrillation $(\mathrm{HR}=$ $0.39 ; 95 \% \mathrm{CI}=0.26-0.59 ; \mathrm{p}<0.0001)$, and after adjustment for mitral regurgitation grade and the presence of a stimulator/cardioverter defibrillator $(\mathrm{HR}=0.35 ; 95 \% \mathrm{CI}=0.23-0.54 ; \mathrm{p}<0.0001)$.

Improved survival was also confirmed in a subset of patients without concomitant left-sided valve defects. The type of valve repair device used (MitraClip vs. other repair systems) did not affect the primary endpoint rate, with MitraClip being the most frequently used device (229 out of 268 patients).

The obvious limitation of the study, in terms of the aim of this paper, is the use of MitraClip rather than TriClip. However, both technologies are based on Alfieri's (edge-to-edge) technique with the identical implants which remains after procedure. Differences between the technologies result from the clip Delivery System and Steering Guide Catheter, reflecting the anatomic challenges of the right side of the heart. Therefore, both technologies can be considered similar enough to allow for the use of the data.

\section{TRILUMINATE study}

The TRILUMINATE study aimed to assess the safety and efficacy of the TriClip TTVr System in the treatment of patients with symptomatic, at least moderate, tricuspid valve insufficiency who were receiving drug therapy and for whom TTVr seemed substantiated [24]. This was a prospective, 
Table 1. TriClip TTVr System effectiveness in the TRILUMINATE study.

\begin{tabular}{|c|c|c|c|}
\hline Endpoint & $\begin{array}{l}\text { Assessment at } \\
30 \text { days }(n=85)\end{array}$ & $\begin{array}{c}\text { Assessment at } \\
6 \text { months }(n=85)\end{array}$ & $\begin{array}{l}\text { Assessment at } \\
1 \text { year }(n=63)\end{array}$ \\
\hline $\begin{array}{l}\text { Reduction in TR severity by at least } \\
1 \text { grade within } 30 \text { days of the procedure }\end{array}$ & $85.5 \%$ & $87.1 \%$ & $87 \%$ \\
\hline NYHA class I/II ( $25.3 \%$ at baseline) & $79.7 \%$ & $86.3 \%$ & $80 \%$ \\
\hline $\begin{array}{l}\text { Mean vena contracta width of TR }[\mathrm{cm}] \\
\text { (1.7 } \mathrm{cm} \text { at baseline) }\end{array}$ & 0.99 & 0.86 & NA \\
\hline \multicolumn{4}{|l|}{ Quality of life endpoints } \\
\hline $\begin{array}{l}6 \mathrm{MWT} \text { [m], mean improvement } \\
(277.6, \mathrm{SD}: 37.1 \text { at baseline) }\end{array}$ & NA & 54.6 (SD: 111.4) & 33.09 (SD: 62.88) \\
\hline $\begin{array}{l}\text { KCCQ, mean improvement } \\
\text { vs. baseline, score }\end{array}$ & 14.2 (SD: 16.7) & 18.6 (SD: 21.5) & 16.81 (SD: 23.4) \\
\hline $\begin{array}{l}\text { SF-36 (MCS), mean improvement } \\
\text { (baseline 44.6, SD: 14.0), score }\end{array}$ & 47.6 (SD: 12.3) & 50.1 (SD: 10.6) & NA \\
\hline $\begin{array}{l}\text { SF-36 (PCS), mean improvement } \\
\text { (35.6, SD: } 9.6 \text { at baseline), score }\end{array}$ & 39.5 (SD: 10.0) & 42.5 (SD: 9.6) & NA \\
\hline
\end{tabular}

6MWT - a 6-minute walk test; KCCQ - Kansas City Cardiomyopathy Questionnaire; MCS — Mental Component Summary; NA — not available; NYHA - New York Heart Association; PCS - Physical Component Summary; SF-36 - Short-Form Health Survey; SD — standard deviation; TR - tricuspid regurgitation

single-arm, multicenter (Europe and the USA) study, with the following inclusion criteria:

- age $\geq 18$ years and $\leq 90$ years at enrolment;

- patients treated according to appropriate standards (including optimal drug therapy) prior to enrolment;

- with moderate-to-severe TR;

- NYHA class II or higher;

- no indications for left-sided heart surgery or mitral valve repair.

Exclusion criteria included:

- PASP above $60 \mathrm{mmHg}$ as estimated by echocardiography;

- prior tricuspid valve procedure or the presence of a cardiac implantable electronic device (e.g., a transvenous stimulation device), which could prevent proper insertion of the TriClip TTVr System device.

The TRILUMINATE study involved 85 patients (66\% females) with co-morbidities and a high surgical risk. The mean patient age was $77.8 \pm$ \pm 7.9 years and the EuroSCORE II score was $8.7 \pm$ $\pm 10.7 \%$. Most patients were diagnosed with severe TR $(84 \%)$, less frequently organic $(12 \%)$ or mixed $94 \%$. The most common co-morbidities included: atrial fibrillation (92\%), hypertension (86\%), kidney diseases (46\%), diabetes mellitus $(22 \%)$ and prior myocardial infarction (18\%). One-third of the patients had undergone mitral valve intervention and $75 \%$ of the patients had NYHA class III/IV disease. The efficacy of device implan- tation (defined as a reduction in TR by at least one grade on echocardiography before discharge) was $91 \%$. After 1 year of follow-up, the reduction in the TR grade (to moderate at most) was reported in $71 \%$ of patients [29]. Clinical improvement in NYHA functional class, a 6-minute walk test, and the quality of life were observed. Overall, postprocedure mortality in 1-year follow-up was $7.1 \%$. Detailed efficacy and safety data for the TriClip TTVr System in the subsequent follow-up period are presented in Tables 1 and 2.

\section{TripClip cost effectiveness}

An attempt was made at estimating the cost-effectiveness of the TriClip TTVr System compared to drug therapy for TR, i.e., assessing a mean difference (calculated per patient) in the cost from the public payer's perspective and the effect expressed by life years gained (LYG) and quality-adjusted life years (QALYs). The analysis used the only comparative study available (and discussed above) [23], so the target population for the analysis was consistent with patient characteristics in the study. A 1-year horizon was used in the study, and the estimated Kaplan-Meier curves for survival at 1 year indicated the value of approximately $77 \%$ for TTVI and $\sim 64 \%$ for drug therapy. Given the difference in survival, benefits from TTVI are also derived in the years following the procedure. To allow for these benefits, the survival curves were 
Table 2. TriClip TTVr System safety in the TRILUMINATE study.

\begin{tabular}{lccc}
\hline Endpoint & $\begin{array}{c}\text { Assessment at } \\
\mathbf{3 0} \text { days }(\mathbf{n}=85)\end{array}$ & $\begin{array}{c}\text { Assessment at } \\
\mathbf{6} \text { months (n=85) }\end{array}$ & $\begin{array}{c}\text { Assessment at } \\
\mathbf{1} \text { year (n = 50) }\end{array}$ \\
\hline Major adverse event & $\mathrm{NA}$ & $5(6 \%)$ & $3(6 \%)$ \\
CV mortality & $2(2.4 \%)$ & $3(3.6 \%)$ & $3(6 \%)$ \\
Myocardial infarction & $0(0 \%)$ & $1(1.2 \%)$ & $0(0 \%)$ \\
Stroke & $0(0 \%)$ & $0(0 \%)$ & $0(0 \%)$ \\
De novo kidney failure & $1(1.2 \%)$ & $1(1.2 \%)$ & $0(0 \%)$ \\
Any CV surgery for device-related AE & $0(0 \%)$ & $0(0 \%)$ & $0(0 \%)$ \\
Major bleeding & $6(7.3 \%)$ & $10(11.9 \%)$ & $7(14 \%)$ \\
Pulmonary thromboembolism & $0(0 \%)$ & $0(0 \%)$ & $0(0 \%)$ \\
Newly diagnosed hepatic failure & $0(0 \%)$ & $0(0 \%)$ & $0(0 \%)$ \\
Newly diagnosed atrial fibrillation & $1(1.2 \%)$ & $1(1.2 \%)$ & $0(0 \%)$ \\
All-cause mortality & $0(0 \%)$ & $4(4.8 \%)$ & $5(10 \%)$ \\
Single device insertion & $\mathrm{NA}$ & $5(7.2 \%)$ & $3(6 \%)$ \\
Embolization & $\mathrm{NA}$ & $0(0 \%)$ & $0(0 \%)$ \\
Tricuspid valve stenosis & $\mathrm{NA}$ & $7(10.8 \%)$ & $\mathrm{NA}$ \\
Tricuspid valve surgery & $\mathrm{NA}$ & $1(1.2 \%)$ & $\mathrm{NA}$ \\
\hline
\end{tabular}

$\mathrm{AE}$ - adverse event; $\mathrm{CV}$ - cardiovascular; $\mathrm{NA}$ - not available

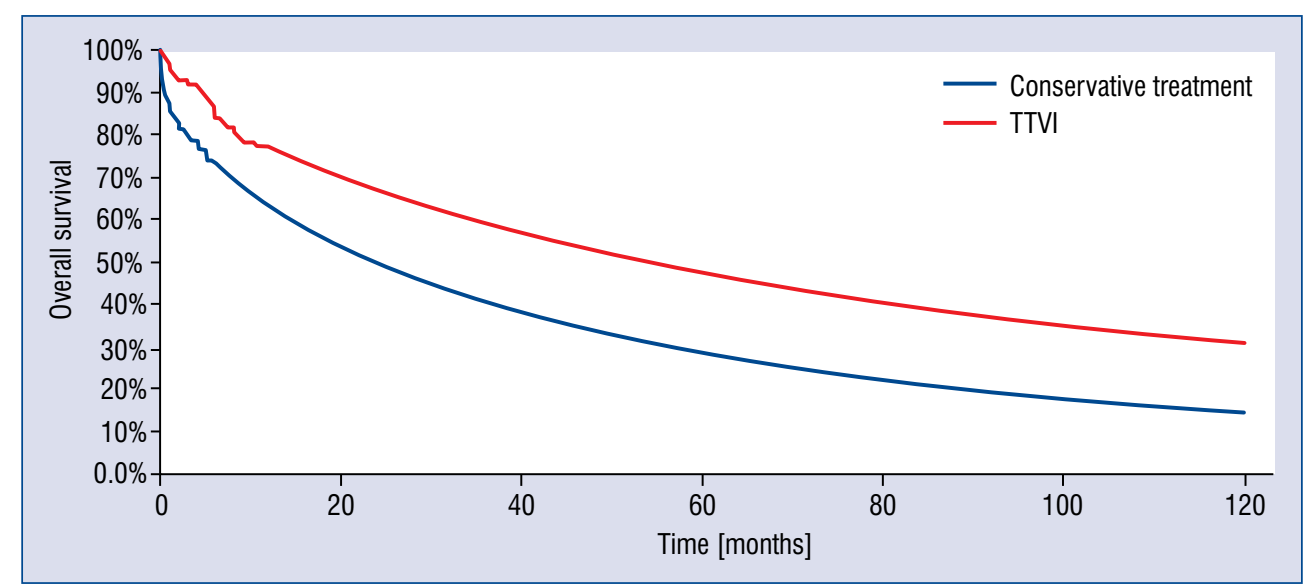

Figure 2. Survival curves for transcatheter tricuspid valve intervention (TTVI) and drug therapy in the 2019 Taramasso study [23], extrapolated using the Weibull model.

extrapolated beyond the horizon of the Taramasso 2019 study to 10 years, using the Weibull model (Fig. 2).

In the analysis of QALYs, information on NYHA class distribution and Kansas City Cardiomyopathy Questionnaire (KCCQ) values at enrolment and at 6 months were used to allow for the technology's impact on health-related quality of life (Table 3). It was assumed that the value of these parameters in patients with the TriClip TTVr System implanted would correspond to those observed 6 months in the study (1-year data were available for an incomplete group of patients). For patients receiving drug therapy, it was assumed that the values would remain at the baseline level. Since only patients on optimal drug therapy at enrolment were included in the TRILUMINATE study, it seems reasonable to assume that NYHA class in the control group should not be subject to significant changes in the short-term horizon. In the long-term horizon, both NYHA and KCCQ may likely worsen in both groups. 
Table 3. New York Heart Association (NYHA) class changes in the TRILUMINATE study (the difference from baseline was statistically significant for 30-day, 6-month and 12-month follow-up, $p<0.0001$ ).

\begin{tabular}{lcccc}
\hline NYHA class & $\begin{array}{c}\text { Assessment at } \\
\text { baseline }(\mathbf{n}=\mathbf{8 3})\end{array}$ & $\begin{array}{c}\text { Assessment at } \\
\mathbf{3 0} \text { days }(\mathbf{n}=\mathbf{8 4})\end{array}$ & $\begin{array}{c}\text { Assessment at } \\
\mathbf{6} \text { months }(\mathbf{n}=\mathbf{7 3})\end{array}$ & $\begin{array}{c}\text { Assessment at } \\
\mathbf{1} \text { year }(\mathbf{n}=\mathbf{6 5})\end{array}$ \\
\hline NYHA I & $0 \%$ & $23 \%$ & $36 \%$ & $32 \%$ \\
NYHA II & $25 \%$ & $57 \%$ & $51 \%$ & $51 \%$ \\
NYHA III & $70 \%$ & $20 \%$ & $12 \%$ & $17 \%$ \\
NYHA IV & $5 \%$ & $0 \%$ & $1 \%$ & $0 \%$ \\
\hline
\end{tabular}

However, from the point of view of the economic analysis results, an intergroup difference in the parameters plays a key role. This paper de facto assumes that the difference is maintained in the long-term horizon.

The NYHA and KCCQ values were assigned health-state utilities based on the results of a systematic literature review. As for KCCQ, 1 study was found [30], which showed the following dependence: utility $=0.44+0.0035 * \mathrm{KCCQ}-\mathrm{OS}$. As for NYHA, several studies were identified and qualified for parameterization, which reported utility values for patients with heart failure in individual NYHA classes [31-36]. Mean utility values for NYHA class I and mean decrements for other groups from the publications found were used. Ultimately, the following utility values were used: 0.858 for NYHA class I, 0.762 for NYHA class II, 0.646 for NYHA class III, and 0.459 for NYHA class IV.

In the analysis, it was assumed that the TTVI cost would amount to approximately PLN 125,000. The costs of rehospitalization were also taken into account, required by $26 \%$ of patients who had received the TriClip TTVr System and 47\% of patients on drug therapy, according to the 2019 Taramasso study. It was assumed that the cost of rehospitalization would equal the weighted average cost of procedure E50: acute or decompensated heart failure - treatment at Intensive Cardiac Care Unit (PLN 17,000; 80\% of patients) and procedure E52: advanced circulatory failure (PLN 5,987; $20 \%$ of patients). The analysis included the additional costs of drug therapy used in both patient groups, amounting to approximately PLN 100 per year. Future costs and effects were discounted at the rates of $5 \%$ and $3.5 \%$, respectively, as per Polish Health Technology Assessment guidelines.

The incremental cost-effectiveness ratio for the TriClip TTVr System compared to drug therapy for TR was PLN 84,109.79/QALY for the analysis based on the NYHA class and PLN 101,290.90/ /QALY for the analysis based on KCCQ. In turn, when considering the impact on life expectancy only, the obtained coefficient amounted to PLN $86,422.02 / \mathrm{LYG}$. In all cases, the incremental cost-effectiveness ratio value was clearly below the cost-effectiveness threshold defined in Poland, i.e., PLN 155,514/QALY (or PLN/LYG).

\section{Estimation of the size of the population that may benefit from the TriClip TTVr System}

As a rule, TriClip TTVr System should be used in patients with TR and a high cardiac surgical risk in whom drug therapy fails to provide satisfactory outcomes. In an attempt to determine the size and structure of this population, four groups may be distinguished, which should constitute the largest part of the target population:

- high surgical risk or inoperable patients with mitral regurgitation and coexistent $\mathrm{TR}$, with indications for the repair of both valves;

- patients after mitral valve surgery who developed moderate-to-severe TR;

- patients with severe TR in the course of RV insufficiency after the implantation of an LV assist device;

- patients with isolated severe TR who, in the opinion of the Heart Team, are not candidates for surgical valve repair/replacement.

The estimation of the size of the first subpopulation was based on determining the annual population of candidates for percutaneous mitral regurgitation treatment. Data from other countries were used for this purpose, as Poland's actual number of procedures may not include all patients who needed it. By calculating the average number of procedures with respect to the number of citizens, the demand for the edge-to-edge procedure in Poland was estimated at 778 per year (due to data confidentiality, they were not presented in this paper). The estimated proportion of patients with additional TR ranges from $19.4 \%$ [37] to $27 \%$ [38]. 
The ultimate size of the first subpopulation can be estimated at approximately 180 patients per year.

According to National Cardiac Surgery Registry data, 896 mitral valve surgeries were performed in Poland in 2019. Assuming that TR develops in $5 \%$ of patients after mitral valve surgery, the size of the second subpopulation can be estimated at 45 per year. According to the authors' clinical experience, the size of the third subpopulation should be estimated at approximately 20 patients per year. Assuming that isolated TR occurs in approximately $8 \%$ of patients [19], the size of the fourth group was estimated with respect to the size of the first three groups, resulting in approximately 20 patients per year.

In total, the number of patients with likely indications for TriClip TTVr System implantation is approximately 265 per year. It should be noted that in the first years of the system's availability, the actual number of procedures would most likely be lower and would reach the estimated level only after a few years.

\section{Summary}

Severe TR leads to heart failure and worsened quality of life and is associated with a poor prognosis. Treatment modalities include drug therapy (of limited effectiveness) and cardiac surgery, associated with a very high risk, especially in patients in a worse clinical condition, with RV dysfunction and co-morbidities. Thanks to the availability of percutaneous treatment of TR, patients may be offered an effective, non-invasive procedure associated with a low risk of complications. The TriClip TTVr System proved safe and effective in the TRILUMINATE study. Based on the Taramasso 2019 study, it may be inferred that transcatheter edge-to-edge tricuspid valve repair is more effective than drug therapy regarding its effect on survival and rehospitalizations but this needs to be confirmed in further studies. In light of the survival benefit, improved quality of life, and anticipated cost, the TriClip TTVr System should be considered cost-effective. A subgroup analysis of patients who could benefit from the technology suggests that the annual number of procedures could be approximately 265 after a few years.

\section{Acknowledgments}

The authors would like to thank Abbott Medical sp. $z$ o.o., Health Economics \& Reimbursement, Warsaw, Poland, for the organization and financial support for the experts' meetings. We would also like to thank HealthQuest employees (I. Lipka and B. Tabor) for their support in preparing parts of this paper.

Conflict of interest: HealthQuest (including Maciej Niewada and Michał Jakubczyk) received fees for preparing materials for the experts' discussion. HealthQuest collaborates with Abbott and receives fees for the preparation of health technology assessments. Adam Witkowski: Abbott Poland Advisory Board member, presentation fees (Abbott); Wojciech Wojakowski: Abbott Poland Advisory Board member, lecture fees (Abbott); Andrzej Gackowski: ECHO proctor - Abbott, Edwards; Marek Grygier: Abbott Poland Advisory Board member, lecture fees, proctor for structural heart interventions (Abbott); Piotr Przygocki: Employee of Abbott.

\section{References}

1. Singh JP, Evans JC, Levy D, et al. Prevalence and clinical determinants of mitral, tricuspid, and aortic regurgitation (the Framingham Heart Study). Am J Cardiol. 1999; 83(6): 897-902, doi: 10.1016/s0002-9149(98)01064-9, indexed in Pubmed: 10190406.

2. Chorin E, Rozenbaum Z, Topilsky Y, et al. Tricuspid regurgitation and long-term clinical outcomes. Eur Heart J Cardiovasc Imaging. 2020; 21(2): 157-165, doi: 10.1093/ehjci/jez216, indexed in Pubmed: 31544933

3. Lindekleiv H, Løchen ML, Mathiesen EB, et al. Echocardiographic screening of the general population and long-term survival: a randomized clinical study. JAMA Intern Med. 2013; 173(17): 1592-1598, doi: 10.1001/jamainternmed.2013.8412, indexed in Pubmed: 23877591.

4. Topilsky Y, Maltais S, Medina Inojosa J, et al. Burden of tricuspid regurgitation in Patients diagnosed in the community setting. JACC Cardiovasc Imaging. 2019; 12(3): 433-442, doi: 10.1016/j. jcmg.2018.06.014, indexed in Pubmed: 30121261.

5. Brykczyński M, Mokrzycki K, Jędrzejczak T. Chirurgiczne leczenie wtórnej niedomykalności zastawki trójdzielnej. Kardiologia po Dyplomie. 2011; 10(4): 82-85.

6. Wang N, Fulcher J, Abeysuriya N, et al. Tricuspid regurgitation is associated with increased mortality independent of pulmonary pressures and right heart failure: a systematic review and meta-analysis. Eur Heart J. 2019; 40(5): 476-484, doi: 10.1093/ eurheartj/ehy641, indexed in Pubmed: 30351406.

7. Nath J, Foster E, Heidenreich PA. Impact of tricuspid regurgitation on long-term survival. J Am Coll Cardiol. 2004; 43(3): 405-409, doi: 10.1016/j.jacc.2003.09.036, indexed in Pubmed: 15013122.

8. Lancellotti P, Tribouilloy C, Hagendorff A, et al. Scientific Document Committee of the European Association of Cardiovascular Imaging. Recommendations for the echocardiographic assessment of native valvular regurgitation: an executive summary from the European Association of Cardiovascular Imaging. Eur Heart J Cardiovasc Imaging. 2013; 14(7): 611-644, doi: 10.1093/ ehjci/jet105, indexed in Pubmed: 23733442.

9. Hahn RT, Zamorano JL. The need for a new tricuspid regurgitation grading scheme. Eur Heart J Cardiovasc Imaging. 2017; 18(12): 1342-1343, doi: 10.1093/ehjci/jex139, indexed in Pubmed: 28977455 . 
10. Dreyfus GD, Martin RP, Chan KM, et al. Functional tricuspid regurgitation: a need to revise our understanding. J Am Coll Cardiol. 2015; 65(21): 2331-2336, doi: 10.1016/j.jacc.2015.04.011, indexed in Pubmed: 26022823.

11. Van Praet KM, Stamm C, Starck CT, et al. An overview of surgical treatment modalities and emerging transcatheter interventions in the management of tricuspid valve regurgitation. Expert Rev Cardiovasc Ther. 2018; 16(2): 75-89, doi: 10.1080/14779072.2018.1421068, indexed in Pubmed: 29283684.

12. Kay JH, Maselli-Campagna G, Tsuji KK. Surgical treatment of tricuspid insufficiency. Ann Surg. 1965; 162: 53-58, doi: 10.1097/00000658-196507000-00009, indexed in Pubmed: 14313519.

13. De Vega NG. [Selective, adjustable and permanent annuloplasty. An original technic for the treatment of tricuspid insufficiency]. Rev Esp Cardiol. 1972; 25(6): 555-556, indexed in Pubmed: 4658611.

14. Baumgartner H, Falk V, Bax JJ, et al. 2017 ESC/EACTS Guidelines for the management of valvular heart disease. Eur Heart J. 2017; 38(36): 2739-2791, doi: 10.1093/eurheartj/ehx391, indexed in Pubmed: 28886619.

15. Otto C, Nishimura R, Bonow R, et al. 2020 ACC/AHA Guideline for the Management of Patients With Valvular Heart Disease: A Report of the American College of Cardiology/American Heart Association Joint Committee on Clinical Practice Guidelines. Circulation. 2021; 143(5), doi: 10.1161/cir.0000000000000923.

16. Konstam MA, Kiernan MS, Bernstein D, et al. Evaluation and management of right-sided heart failure: a scientific statement from the American Heart Association. Circulation. 2018; 137(20): e578-e622, doi: 10.1161/CIR.0000000000000560, indexed in Pubmed: 29650544.

17. Chang CC, Veen KM, Hahn RT, et al. Uncertainties and challenges in surgical and transcatheter tricuspid valve therapy: a stateof-the-art expert review. Eur Heart J. 2020; 41(20): 1932-1940, doi: 10.1093/eurheartj/ehz614, indexed in Pubmed: 31511897.

18. Kilic A, Saha-Chaudhuri P, Rankin JS, et al. Trends and outcomes of tricuspid valve surgery in North America: an analysis of more than 50,000 patients from the Society of Thoracic Surgeons database. Ann Thorac Surg. 2013; 96(5): 1546-52; discussion 1552, doi: 10.1016/j.athoracsur.2013.06.031, indexed in Pubmed: 24070702.

19. Dreyfus J, Flagiello M, Bazire B, et al. Isolated tricuspid valve surgery: impact of aetiology and clinical presentation on outcomes. Eur Heart J. 2020; 41(45): 4304-4317, doi: 10.1093/ eurheartj/ehaa643, indexed in Pubmed: 32974668.

20. Oliveira DC, Oliveira CGC. The forgotten, not studied or not valorized tricuspid valve: the transcatheter revolution is coming. Cardiol Res. 2019; 10(4): 199-206, doi: 10.14740/cr874, indexed in Pubmed: 31413775.

21. Taramasso M, Hahn RT, Alessandrini H, et al. The international multicenter trivalve registry: which patients are undergoing transcatheter tricuspid repair? JACC Cardiovasc Interv. 2017; 10(19): 1982-1990, doi: 10.1016/j.jcin.2017.08.011, indexed in Pubmed: 28982563.

22. Mehr M, Taramasso M, Besler C, et al. 1-Year outcomes after edge-to-edge valve repair for symptomatic tricuspid regurgitation: results from the TriValve registry. JACC Cardiovasc Interv. 2019; 12(15): 1451-1461, doi: 10.1016/j.jcin.2019.04.019, indexed in Pubmed: 31395215.

23. Taramasso M, Benfari G, van der Bijl P, et al. Transcatheter versus medical treatment of patients with symptomatic severe tricuspid regurgitation. J Am Coll Cardiol. 2019; 74(24): 2998-3008, doi: 10.1016/j.jacc.2019.09.028, indexed in Pubmed: 31568868.

24. Nickenig G, Weber M, Lurz P, et al. Transcatheter edge-toedge repair for reduction of tricuspid regurgitation: 6-month outcomes of the TRILUMINATE single-arm study. Lancet. 2019;
394(10213): 2002-2011, doi: 10.1016/S0140-6736(19)32600-5, indexed in Pubmed: 31708188.

25. Rosenbaum P, Rubin D. The central role of the propensity score in observational studies for causal effects. Biometrika. 1983; 70(1): 41-55, doi: 10.1093/biomet/70.1.41.

26. Blackstone EH. Breaking down barriers: helpful breakthrough statistical methods you need to understand better. J Thorac Cardiovasc Surg. 2001; 122(3): 430-439, doi: 10.1067/ mtc.2001.117536, indexed in Pubmed: 11547291.

27. Blackstone EH. Comparing apples and oranges. J Thorac Cardiovasc Surg. 2002; 123(1): 8-15, doi: 10.1067/mtc.2002.120329, indexed in Pubmed: 11782750.

28. Brookhart MA, Wyss R, Layton JB, et al. Propensity score methods for confounding control in nonexperimental research. Circ Cardiovasc Qual Outcomes. 2013; 6(5): 604-611, doi: 10.1161/ CIRCOUTCOMES.113.000359, indexed in Pubmed: 24021692.

29. Lurz P, Stephan von Bardeleben R, Weber M, et al. Transcatheter edge-to-edge repair for treatment of tricuspid regurgitation. J Am Coll Cardiol. 2021; 77(3): 229-239, doi: 10.1016/j. jacc.2020.11.038, indexed in Pubmed: 33478646.

30. Goates S, Baron SJ, Arnold SV, et al. Estimating health-state utility in patients with heart failure: mapping the kansas city cardiomyopathy questionnaire to SF-6D utility scores. Value in Health. 2019; 22: S124, doi: 10.1016/j.jval.2019.04.466.

31. Alehagen U, Rahmqvist M, Paulsson T, et al. Quality-adjusted life year weights among elderly patients with heart failure. Eur J Heart Fail. 2008; 10(10): 1033-1039, doi: 10.1016/j.ejheart.2008.07.015, indexed in Pubmed: 18760669.

32. Berg J, Lindgren P, Mejhert M, et al. Determinants of utility based on the EuroQol Five-Dimensional Questionnaire in patients with chronic heart failure and their change over time: results from the swedish heart failure registry. Value Health. 2015; 18(4): 439-448, doi: 10.1016/j.jval.2015.02.003, indexed in Pubmed: 26091598.

33. Göhler A, Geisler BP, Manne JM, et al. Utility estimates for decision-analytic modeling in chronic heart failure: health states based on New York Heart Association classes and number of rehospitalizations. Value Health. 2009; 12(1): 185-187, doi: 10.1111/j.15244733.2008.00425.x, indexed in Pubmed: 18647251.

34. Griffiths A, Paracha N, Davies A, et al. Analyzing health-related quality of life data to estimate parameters for cost-effectiveness models: an example using longitudinal EQ-5D data from the SHIFT randomized controlled trial. Adv Ther. 2017; 34(3): 753-764, doi: 10.1007/s12325-016-0471-x, indexed in Pubmed: 28205056.

35. Kirsch J, McGuire A. Establishing health state valuations for disease specific states: an example from heart disease. Health Econ. 2000; 9(2): 149-158, doi: 10.1002/(sici)10991050(200003)9:2<149::aid-hec501>3.0.co;2-n, indexed in Pubmed: 10721016 .

36. Linde C, Mealing S, Hawkins N, et al. REVERSE study group. Cost-effectiveness of cardiac resynchronization therapy in patients with asymptomatic to mild heart failure: insights from the European cohort of the REVERSE (Resynchronization Reverses remodeling in Systolic Left Ventricular Dysfunction). Eur Heart J. 2011; 32(13): 1631-1639, doi: 10.1093/eurheartj/ehq408, indexed in Pubmed: 21112898.

37. Ejiofor JI, Hirji SA, Ramirez-Del Val F, et al. Outcomes of repeat mitral valve replacement in patients with prior mitral surgery: A benchmark for transcatheter approaches. J Thorac Cardiovasc Surg. 2018; 156(2): 619-627.e1, doi: 10.1016/j.jtcvs.2018.03.126, indexed in Pubmed: 29759741.

38. Pręgowski J, Kłapyta A, Chmielak Z, et al. Incidence, clinical correlates, timing, and consequences of acute thrombus formation in patients undergoing the MitraClip procedure. Kardiol Pol. 2020; 78(1): 45-50, doi: 10.33963/KP.15056, indexed in Pubmed: 31719512 . 